\title{
LONG TERM OUTCOME FROM UNILATERAL ADRENALECTOMY IN PATIENTS WITH PRIMARY ALDOSTERONISM
}

\author{
${ }^{1}$ Hannon MJ, ${ }^{1}$ Sze WC, ${ }^{2}$ Carpenter R, 2 Parvanta L, ${ }^{3}$ Matson M, \\ ${ }^{3}$ Sahdev A, ${ }^{1}$ Druce MR, ${ }^{1}$ Waterhouse M, ${ }^{1}$ Akker SA, ${ }^{1}$ Drake WM \\ Departments of ${ }^{1}$ Endocrinology, ${ }^{2}$ Surgery, ${ }^{3}$ Radiology \\ St. Bartholomew's Hospital, West Smithfield, London, UK
}

\section{Background:}

Primary aldosteronism (PA) is the most common cause of secondary hypertension

It affects $\mathbf{2 0 - 2 5 \%}$ of those with treatment resistant hypertension

If left untreated it has a significant cardiometabolic risk over and above that conferred by hypertension alone

$\mathbf{5 0 \%}$ are due to a unilateral aldosterone producing adrenal adenoma and therefore curable with unilateral adrenalectomy

However, the number of adrenalectomies in the UK falls far short of this because:

\section{- $\quad$ lack of access to adrenal venous sampling}

sparse long term outcome data

This study examines long term outcomes in patients undergoing unilateral adrenalectomy for PA

Methods

Retrospective case notes review

120 consecutive patients, 51 underwent unilateral adrenalectomy

Follow up data gathered at least 3 months after surgery:

- Resting supine blood pressure (outpatients' clinic)

- Home blood pressure readings

- Number of antihypertensive drugs pre and post operatively

- Renin and aldosterone measurements

- Renin/aldosterone measured in the absence of interfering medications

Results:

31 males, 20 females, median age 54 (range 30 - 83)

Median follow up 48 months (range $30-154$ )

$23 / 51(45.1 \%)$ had "cured" hypertension (not on antihypertensives post-op)

$25 / 51$ (49\%) had improved hypertension (still requiring antihypertensives post-op)

\section{Results:}

Overall results for all patients:

\begin{tabular}{|c|c|c|c|}
\hline Variable & Pre-Op & Post-Op & $p$ value \\
\hline BP & $\begin{array}{c}160 / 95 \\
(120 / 80- \\
250 / 150)\end{array}$ & $\begin{array}{c}130 / 80 \\
(110 / 70- \\
160 / 93)\end{array}$ & $<0.0001$ \\
\hline $\begin{array}{c}\text { No. of } \\
\text { antihypertensives } \\
\text { Median (range) }\end{array}$ & 3 & 1 & $<0.0001$ \\
\hline $\begin{array}{c}\text { Serum [K+] } \\
\text { mmol/L }\end{array}$ & 3.2 & 4.4 & $<0.0001$ \\
Median (range) & $(2.3-4.7)$ & $(3.3-5.3)$ & \\
\hline
\end{tabular}

Results for patients improved but not cured:

\begin{tabular}{|c|c|c|c|}
\hline Variable & Pre-Op & Post-Op & $p$ value \\
\hline $\begin{array}{c}\text { BP } \\
\text { Median (range) }\end{array}$ & $\begin{array}{c}160 / 98 \\
(140 / 80- \\
250 / 150)\end{array}$ & $\begin{array}{c}130 / 80 \\
(120 / 70- \\
169 / 93)\end{array}$ & 0.0004 \\
\hline $\begin{array}{c}\text { No. of } \\
\text { antihypertensives } \\
\text { Median (range) }\end{array}$ & 3 & 1 & $<0.0001$ \\
\hline $\begin{array}{c}\text { Serum [K } \\
\text { mmol/L } \\
\text { Median (range) }\end{array}$ & 3.2 & $(0-3)$ & \\
\hline & $(2.4-4.7)$ & $(3.5-5.3)$ & $<0.0001$ \\
\hline
\end{tabular}

More females in "cured" group:

$.17 / 23$ vs $4 / 25, p<0.0001$

No other differences between those "cured" and those improved but not cured in terms of age, pre-op BP, preop $\left[\mathrm{K}^{+}\right]$, number of pre-op antihypertensives, or size of site of excised adenoma

$3 / 51$ (5.9\%) had no improvement in BP post-op

-Histology in these 3 patients showed typical adenoma in each case

\section{Discussion and Conclusions}

Laparoscopic-assisted adrenalectomy provides excellent long term improvements in blood pressure, hypokalaemia and polypharmacy

These data should inform discussions with patients regarding surgical outcomes 